\title{
Waveguide based optofluidics
}

\author{
Genni Testa ${ }^{\mathrm{a}}$, Yujian Huang ${ }^{\mathrm{c}}$,Luigi Zeni ${ }^{\mathrm{b}}$, Pasqualina M. Sarro ${ }^{\mathrm{c}}$, Romeo Bernini ${ }^{\mathrm{a}^{*}}$ \\ ${ }^{a}$ IREA-CNR,Via Diocleziano 328, 80124 Napoli, Italy \\ ${ }^{b}$ DII, Seconda Università di Napoli, Via Roma 29, 81031 Aversa. \\ 'ECTM-DIMES, TUDelft, Feldmannweg 17, NL-2600 GB Delft, The Netherlands.
}

\begin{abstract}
Integrated liquid core AntiResonant Reflecting Optical Waveguide (ARROW) are used as basic component for the realization of complex optofluidic devices. Liquid core ARROW waveguides permit to confine the light in a low refractive index liquid core, by means of two high refractive index cladding layers designed to form a high reflectivity Fabry-Perot antiresonant cavity. This arrangement allows to realize liquid core waveguides that can be very useful in optofluidic applications. We report the fabrication and the characterization different optofluidic devices based on hollow core ARROW waveguide like tuneable couplers and Mach-Zehnder interferometers. The proposed devices have been realized by silicon technology. The channels have been realized by etching the silicon wafer, while the two claddings have been deposited on both wafers by LPCVD or ALD depositions.
\end{abstract}

Keywords: Optofluidics, Liquid core waveguides, Integrated silicon devices.

\section{INTRODUCTION}

Optofluidics is a very promising field that has seen a significant improvement over the last few years. Optofluidics is essentially the integration of optics and microfluidics [1]. This permits to realize innovative optical system in which the fluids can be used to efficiently change the optical properties of a device.

In this field liquid core waveguides can represent an important component of future optofluidic devices and systems [2]. In fact they represent the maximum integration and functionality between waveguides, the key element of the optical structure, and microchannels, the key element of microfluidics.

Many techniques have been proposed in order to realize liquid core waveguides. Metallic walls hollow waveguides have been largely studied, however the propagation loss of these waveguides can be very large at certain optical frequencies [3]. Teflon coated waveguides have been proposed and fabricated by means of integrated planar technology [4]. Recently, a dielectric cladding optical waveguide based on bragg reflectors or photonic band gap has been proposed and fabricated [5]. However, its fabrication as integrated device is not easy and requires high index contrast between the cladding layers, which typically necessitates the use of high-index materials like silicon that is absorbing in the visible range, so limiting the range of potential applications. A different way to realize hollow optical waveguide is the use of leaky waveguide [6] such as AntiResonant Reflecting Optical Waveguide (ARROW). Differently from conventional waveguides, in ARROW waveguides the field is not confined in the core region by total internal reflection but by dielectric cladding layers designed to form high reflectivity Fabry-Perot mirrors [7]. This peculiar structure offers some unique properties that make it very attractive for photonics integrated circuits with applications ranging from telecommunications to sensors. In particular in ARROW waveguides the core has a refractive index lower the one of the cladding layers, permitting the realization of liquid core waveguides without metal walls [7-12]. Even though the ARROW modes are leaky, low-loss propagation over large distances can be achieved. Furthermore, singlemode waveguides can be realized also with large core dimensions. These waveguides can be easily designed using very simply optical equations that take into account the interference phenomena in the cladding layers.

In this work, we show that liquid core ARROW waveguide can be used as a basic component for complex integrated optofluidic devices. In particular, we report the fabrication and the characterization of a tunable couplers and two MachZehnder interferometers.

*bernini.r@irea.cnr.it, phone+39081570799, fax:+390815705734, www.irea.cnr.it. 


\section{INTEGRATED LIQUID CORE ARROW WAVEGUIDES}

The transverse section of an integrated liquid core ARROW waveguide is shown in Fig. 1. Two halves compose the waveguide. On both halves, two dielectric layers are deposited on a silicon substrate, having refractive index and thickness $\mathrm{n}_{2}, \mathrm{~d}_{2}$, and $\mathrm{n}_{1}, \mathrm{~d}_{1}$, respectively. In this waveguide, the light is confined inside the core region, where the refractive index $n_{c}$ is lower than the one of the surrounding media, by the two cladding layers designed to form a high reflectivity Fabry-Perot antiresonant cavity.

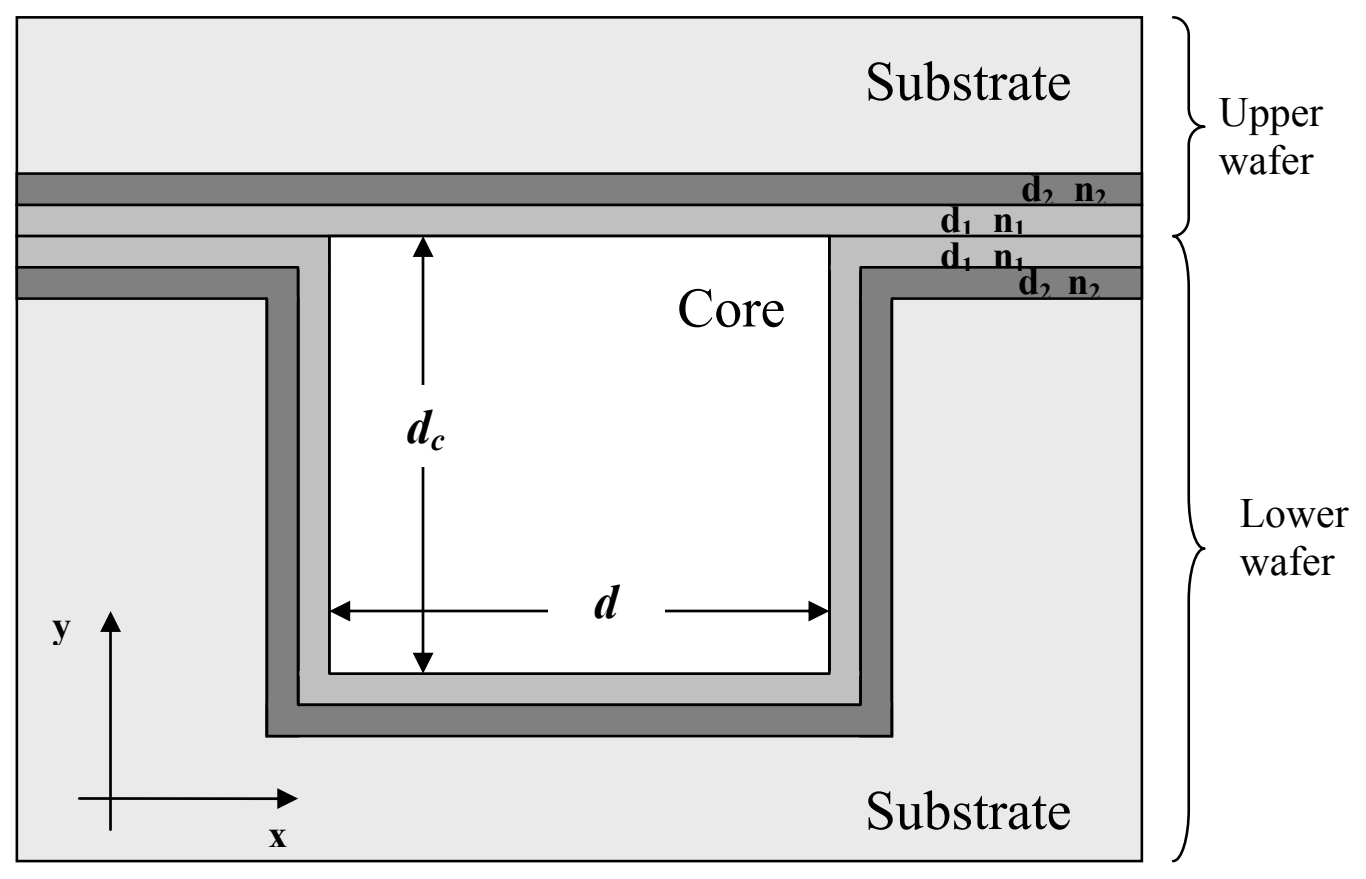

Fig.1 Liquid core ARROW waveguide transverse section.

For a fixed core dimension $d_{c}$ and refractive index $n_{c}$, the antiresonant condition for the equivalent one-dimensional vertical structure permits to calculate the optimal thicknesses of the two cladding layers, as [7]:

$$
\mathrm{d}_{1,2}=\frac{\lambda}{4}\left[\mathrm{n}_{1,2}^{2}-\mathrm{n}_{\text {eff }}^{2}\right]^{\frac{-1}{2}}(2 \mathrm{~N}+1) \quad \mathrm{N}=0,1,2, \ldots
$$

where, $\lambda$ is the working wavelength and $\mathrm{n}_{\text {eff }}$ is the effective index of the propagation mode and the integer $\mathrm{N}=0,1,2, \ldots$ is the antiresonance order. The order of antiresonance determines the width of the transmitted spectrum [13]. In order to have waveguides with a broad-band output transmission spectrum it is necessary to set $\mathrm{N}=0$ in the equation (1) for each cladding layer.

For the fundamental mode $\mathrm{n}_{\text {eff }}$ can be approximately given by [7]:

$$
\mathrm{n}_{\text {eff }}=\frac{\beta}{\beta_{0}} \cong \mathrm{n}_{\mathrm{c}}\left[1-\left(\frac{\lambda}{2 \mathrm{n}_{\mathrm{c}} \mathrm{d}_{\mathrm{c}}}\right)^{2}\right]^{\frac{1}{2}}
$$

where $\beta_{0}=2 \pi / \lambda, \beta$ is propagation constant and $\mathrm{d}_{\mathrm{c}}$ is the thickness of the core.

If $\lambda / \mathrm{dc}<<1$ (e.g. large core waveguides) eq. (2) can be simplified to neff $\cong \mathrm{nc}$. 
ARROWs are leaky waveguides and so the design of an ARROW waveguide requires a special attention depending on their application. In particular, single mode behaviour is an important requirement for optical waveguide devices for use with single-mode fiber or for the fabrication of interferometric devices. With a suitable design large core waveguide with virtual mono-mode behaviour, where higher order modes are filtered out by loss discrimination due to the low reflectivity at the cladding layers for these modes, can be achieved. So, the core dimension of a single mode ARROW waveguides results from a trade-off between the quasi single mode behaviour and the fundamental mode losses. For liquid core waveguide $(\mathrm{nc}=1.330)$ in order to ensure this condition the core dimension has been fixed to be $10 \mathrm{x} 5 \mu \mathrm{m}^{2}$ [14]. The choice of a rectangular core is related to the strong polarization dependence of the losses in an ARROW waveguide. In fact, it is well known that the attenuation for TE polarization is always lower compared with TM for the same core thickness [15]. Since a TE polarized input light can be regarded as TE for transverse confinement and TM for lateral confinement, the $\mathrm{x}$ dimension of the core has to be wider to keep the TM-like loss in this direction low.

\subsection{ARROW fabrication}

ARROW waveguides can be easily fabricated thanks to the well-established silicon technology processes [10-15]. To confine the light, a multilayer stack must be realized by alternating high index contrast cladding layers surrounding the core. Each cladding layer is design to act as a Fabry-Perot (F-P) mirror at a designed wavelength according eq.(1). For the fabrication of these waveguides, material cladding such as silicon nitride $(\mathrm{n}=2.01-2.2)$ and silicon dioxide $(\mathrm{n}=1.46)$ have been widely used as they provide the requirement of a CMOS compatible process and high index contrast. Depositions are generally carried out using Plasma-Enhanced Chemical Vapour Deposition (PECVD) or low pressure chemical vapour deposition (LPCVD) $[10,14]$.

In our case, the fabrication process of an ARROW waveguide starts with the realization of the microchannels by means of a $5 \mu \mathrm{m}$ depth silicon dry etching on the bottom wafer. After that, an LPCVD deposition at a temperature of $850^{\circ} \mathrm{C}$, on both wafers of the silicon dioxide (TEOS) $\left(\mathrm{n}_{2}=1.457\right)$ and silicon nitride $\left(\mathrm{n}_{1}=2.227\right)$ cladding layers, has been realized. The cladding layers thicknesses are $\mathrm{d}_{1}=266 \mathrm{~nm}(\mathrm{~N}=1)$ and $\mathrm{d}_{2}=266 \mathrm{~nm}(\mathrm{~N}=0)$, according to the antiresonant condition (1) at $\lambda=633 \mathrm{~nm}$ and $\mathrm{n}_{\mathrm{c}}=1.330$.

As reported in the previous section, in order to have waveguides with a broad-band output transmission spectrum it should be to set $\mathrm{N}=0$ in the eq.(1) for each cladding layer. However, the choice of the silicon nitride as the first cladding material, depending on the designed wavelength $\lambda$ and on the core refractive index, results in a layer thickness $d_{1}$ ranging from 80 to $100 \mathrm{~nm}(\mathrm{~N}=0$ and $\lambda$ in the visible range of the spectrum). While the deposition of thicker film can be readily achieved by using the PECVD technique, the fabrication of very thin layer can be problematic. Moreover, this deposition process is characterized by a step coverage $\neq 1$, meaning that the vertical cladding layers will result thinner $(\sim 25-30 \%)$ than the horizontal ones [15]. This effect is intrinsic to the PECVD deposition process and it causes that the vertical layers do not fulfil the antiresonance condition (1), in this way leading to an increasing of the overall propagation losses in the waveguide. These factors could represent a relevant drawback in the fabrication of ARROW waveguides for sensing applications. The low pressure chemical vapour deposition (LPCVD) technique, processing at much lower pressure than PECVD, is often used in order to achieve a better conformality of the deposited film. However, conformality and film thicknesses uniformity over large area, complex-shaped substrates, are hard to obtain even with such a deposition technique.

These limitations can be overcome by using Atomic Layer Deposition (ALD) process. The ALD process is a selflimiting process with an average deposition rate very low (usually less than a nanometer per cycle). One of the most important advantages of this technique is that it allows the deposition of very thin cladding layer (sub-100nm) with an excellent conformality, reproducibility and with a high precision over the resulting thickness [16]. Furthermore, this technique permits to considerably reduce the surface roughness, a suitable aspect for optical employment as it allows a strong reduction of optical scattering.

Using ALD ARROW waveguide were fabricated by alternating layer of silicon dioxide and titanium dioxide ( $\mathrm{n}=2.49$ ), where the deposition of the titanium dioxide is realized by ALD deposition technique. $\mathrm{The}^{\mathrm{TiO}} \mathrm{T}_{2}$ layer thickness is $\mathrm{d}_{1}=75.4 \mathrm{~nm}(\mathrm{~N}=0)$. Among the possible dielectric materials that can be deposited by ALD (i.e. TiO2, Al2O3, MgO), we have chosen the titanium dioxide as it provides the higher index contrast with the $\mathrm{SiO}_{2}$. The $\mathrm{TiO}_{2}$ film deposition was carried out by alternately exposing the substrate to volatilized $\mathrm{TiCl}_{4}$ and $\mathrm{H}_{2} \mathrm{O}$ as the precursor of titanium and oxygen, respectively. The precursors are introduced into the chamber with $\mathrm{N}_{2}$ as carrier gas, which is also used to purge the chamber from the excess reaction product. The cycle time for the precursor is $1 \mathrm{~s}$ while the purge period between reactant pulses was $2 \mathrm{~s}$ after $\mathrm{TiCl}_{4}$ exposition and $3 \mathrm{~s}$ after $\mathrm{H}_{2} \mathrm{O}$ exposition. The film was growth at a temperature of $\mathrm{T}=300^{\circ} \mathrm{C}$ with 
a deposition rate of $0.56 \AA$ /cycle. These process parameters were previously optimized in order to obtain a step coverage of $\sim 1$. The thickness distribution of the produced layer was measured with a spectroscopic ellipsometer, resulting in a root mean square (rms) value of the of $0.3 \mathrm{~nm}$ on the wafer area.

The waveguide exhibits a fundamental waveguide loss of $5.22 \mathrm{~cm}^{-1}$ that is in a good agreement with the simulated one $5.11 \mathrm{~cm}^{-1}$. These results confirm that the ALD process for realizing the first cladding layer of a liquid core ARROWs permits to obtain an optimized structure with very good optical performances [13].

\section{ARROW WAVEGUIDE BASED OPTOFLUIDIC DEVICES}

In this section, we report three optofluidic devices based on liquid core ARROW waveguide. A 2 X2 multimode interference coupler and two Mach Zehnder interferometers.

\subsection{ARROW-BASED $2 \times 2$ MMI COUPLER}

Integrated multimode interference (MMI) devices have become very useful and popular due to their excellent performances such as large optical bandwidth, compactness, and relatively easy fabrication [17]. MMI devices are based on the self-imaging property of multimode propagation in waveguide. However, in most applications MMI operates as passive component and only few examples of their use as active component has been investigated. In particular, tuneable devices have been realized in semiconductor based solid core waveguides by localized refractive index changes in the MMI region by current injection. As concerning hollow core waveguides, examples of optical switch and filter based on mechanical tuning of MMI geometry have been proposed [18-20].

Here we present the fabrication and the characterization of a $2 \times 2$ optofluidic multimode interference coupler based on a liquid core antiresonant reflecting optical waveguide (ARROW). In the proposed devices the refractive index of liquid core can be continuously changed by replacing the fluids within the core. In this way, the optical properties of the device can be easily tuned, allowing for the realization of a reconfigurable MMI coupler.

In Fig. 2 the schematic layout of the proposed $2 \times 2$ MMI coupler is depicted. The two input (IP1 and IP2) and output ports (OP1 and OP2) of the MMI device are monomodal ARROW waveguides with the same fundamental mode $\psi_{\mathrm{i}}$ while the MMI region is designed to perform a single mode operation in the vertical transverse dimension (y direction) and a multimode operation in the other (x). The theoretical analysis of the self-imaging will thus be investigated by considering the $1 \mathrm{D}$ structure in the $\mathrm{x}$ direction.

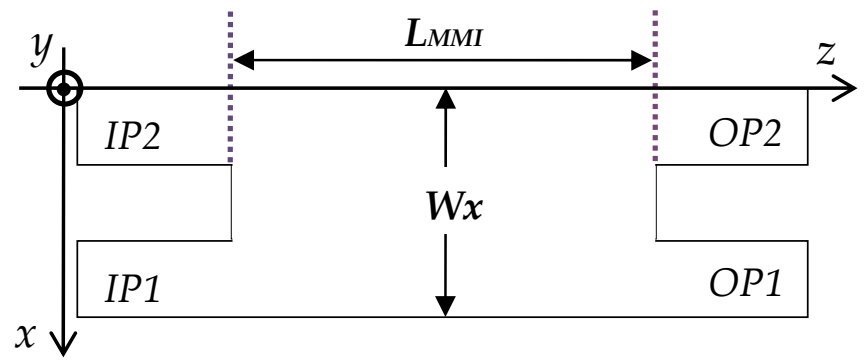

Fig.2 Schematic top view of the proposed $2 \times 2$ MMI coupler.

By defining $\mathrm{L}_{\pi}$ as the beating length of the two lowest-order modes [17]:

$$
\mathrm{L}_{\pi}=\frac{\pi}{\beta_{0}-\beta_{1}} \approx \frac{4 \mathrm{n}_{\mathrm{c}} \mathrm{W}_{\mathrm{x}}^{2}}{3 \lambda}
$$

The minimum distance at which we get the mirrored image of the input field occurs is [17]:

$$
L=\frac{4 n_{c} W_{x}^{2}}{\lambda}
$$


The proposed configuration has $\mathrm{L}_{\mathrm{MMI}}=\mathrm{L}$ so to reproduce an input field on the IP1 (IP2) in a mirrored single image on the OP2 (OP1) by means of the multimodal interference.

The device exhibits interesting spectral filtering properties related to the wavelength dependence of self-imaging principle [20]. In fact, the transmitted intensity at a fixed wavelength, depends on the overlap between the field of the multimode ARROW waveguide at $\mathrm{z}=\mathrm{L}$ and the modal field of the output waveguides. The maximum overlap is reached at the wavelength $\lambda=4 \mathrm{n}_{\mathrm{c}} \mathrm{W}_{\mathrm{x}}^{2} / \mathrm{L}$ (see eq. (4)) for which a single replica of the input field is obtained. Whereas, at different wavelengths the coupling coefficient drops. Furthermore, for a fixed the length $L$ and width $\mathrm{W}_{\mathrm{x}}$ of the MMI region, the wavelength that fulfill the self-imaging condition depends on the refractive index $n_{c}$ of liquid core. This implies that these filtering properties can be easily tuned by simply exchanging the fluids within the core.

The device has been fabricated by LPCVD deposition. The input and output waveguides have a core dimension of $10 \mathrm{x} 5 \mu \mathrm{m}^{2}$. The width of the MMI region is $\mathrm{W}_{\mathrm{x}}=29.25 \mu \mathrm{m}$. Assuming as refractive index for the liquid core $\mathrm{n}_{\mathrm{c}}=1.325$ (methanol), the length of the MMI region resulting from eq. (4) is $\mathrm{L}_{\mathrm{MMI}}=\mathrm{L} \sim 7557 \mu \mathrm{m}$. The design wavelength is $\lambda=600 \mathrm{~nm}$. In Fig. 3 a scanning electron microscope (SEM) picture of the bottom wafer of the fabricated devices is illustrated.

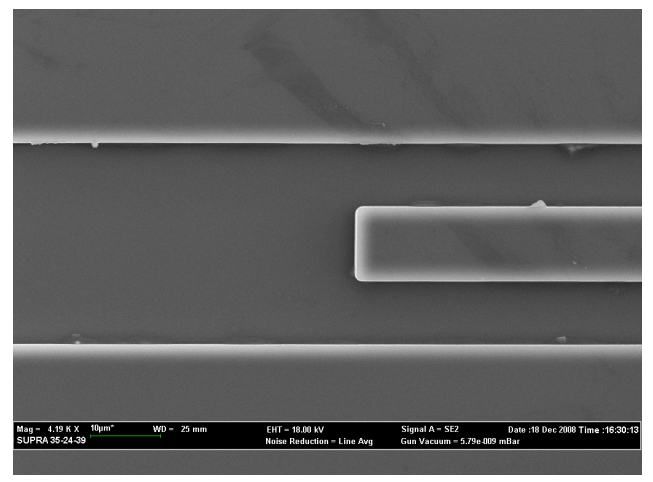

Fig.3 SEM picture of the bottom wafer of the fabricated devices.

The fabricated device has been characterized by coupling an unpolarized light from a white lamp into the input waveguide (IP2) using an optical fiber $(10 \mu \mathrm{m}$ core diameter). A collecting fiber with a core diameter of $10 \mu \mathrm{m}$ has been used for the transmission measurements. The transmitted light has been sent to a CCD spectrometer (range $\lambda=350$ to $800 \mathrm{~nm}$ ) for the spectral characterization. The recorded spectra have been normalized to white lamp spectrum.

In Fig.4 the experimental results for the MMI filled with methanol (MMIm)are shown. The spectra are normalized by the larger value of the maxima of two curves. As expected, the device demonstrates spectral filtering properties that can be explained taking into account the self-imaging principle and the ARROW structure.

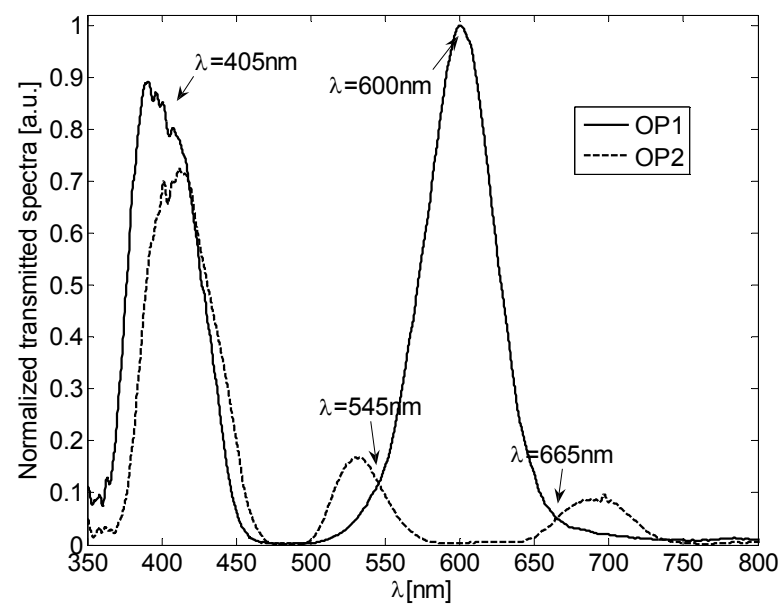

Fig.4 Measured transmitted spectra from OP1 (Solid Line) and from OP2 (Dashed line) of the MMI methanol-filled device. 
From the measured spectra we have estimated an extinction ratio of $\sim 25 \mathrm{~dB}$ between the two output waveguides at $\lambda \sim 600 \mathrm{~nm}$. Whereas, the device behaves as a power splitter at $\lambda=405 \mathrm{~nm}, \lambda=545 \mathrm{~nm}$ and $\lambda=665 \mathrm{~nm}$. From these results we can conclude that at $\lambda \sim 600 \mathrm{~nm}$ the replica of the input field is obtained on the OP1.

In Fig.5 are illustrated the measured transmitted spectra from the two output ports for the ethanol-filled device (MMIe). The results show essentially an overall red-shift of the spectra in comparison with the methanol-filled devices. This shift is related to self-imaging effect and not to the ARROW structure that exhibits a blue-shift as the refractive index of the core increase [11]. In this configuration the device shows splitting capability at $\lambda \sim 405 \mathrm{~nm}$ and $\lambda \sim 563 \mathrm{~nm}$, while at $\lambda \sim 610 \mathrm{~nm}$ the device behaves as a filter, with an extinction ratio of $\sim 23 \mathrm{~dB}$.

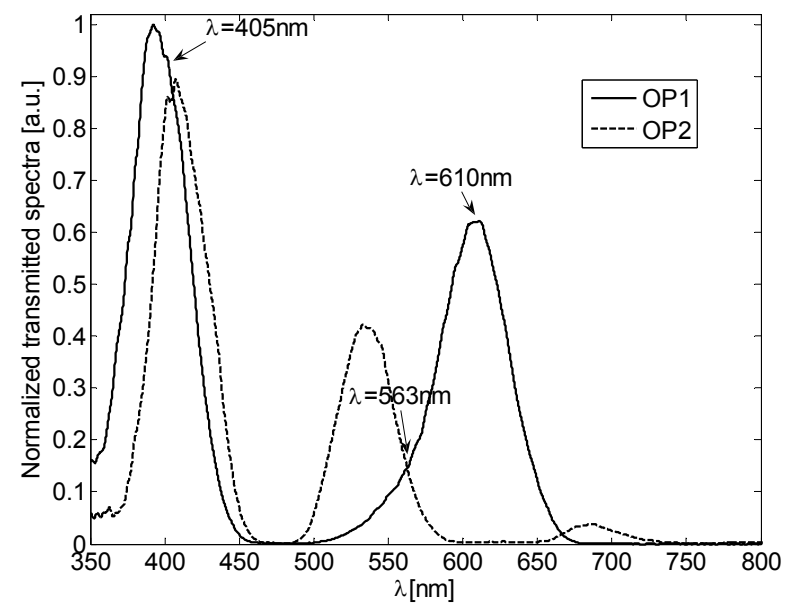

Fig.5 Measured transmitted spectra from OP1 (Solid Line) and from OP2 (Dashed line) of the MMI ethanol-filled device.

In order to highlight the tunability of the device as a function of the refractive index core, in Fig.6 the spectra transmitted from the MMIm and the MMIe has been shown. As can be observed, at $\lambda=545 \mathrm{~nm}$ the ratio between the transmitted intensity from the two output waveguides of the MMIm, measured from the spectra, is $\left(\mathrm{I}_{1} / \mathrm{I}_{2}\right)_{\mathrm{m}} \sim 1$ while for the MMIe at the same wavelength is $\left(\mathrm{I}_{1} / \mathrm{I}_{2}\right)_{\mathrm{e}} \sim 0.2$. At $\lambda=563 \mathrm{~nm}$, conversely, is $\left(\mathrm{I}_{1} / \mathrm{I}_{2}\right)_{\mathrm{e}} \sim 1$ and $\left(\mathrm{I}_{1} / \mathrm{I}_{2}\right)_{\mathrm{m}} \sim 9$. These results show that the power splitting ratio into the two output ports can be tuned in a large range by simply replacing the fluid filling the core.

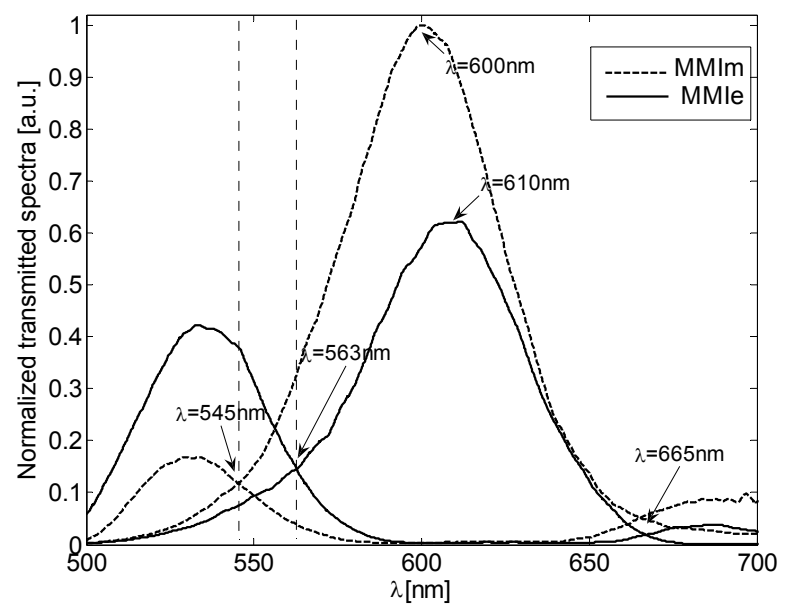

Fig.6 Measured transmitted spectra of the MMIm device (Dashed Line) and MMIe device (Solid line).

In order to fully evaluate the splitting performance of the device, we have captured the images of the field profile transmitted from the output waveguides at different excitation wavelengths. The transmitted intensities have been imaged through a microscope objective on a CCD camera. The recorded direct output images from MMIm and MMIe at 
$\lambda=545 \mathrm{~nm}$ and $\lambda=563 \mathrm{~nm}$ are reported in figure 7.

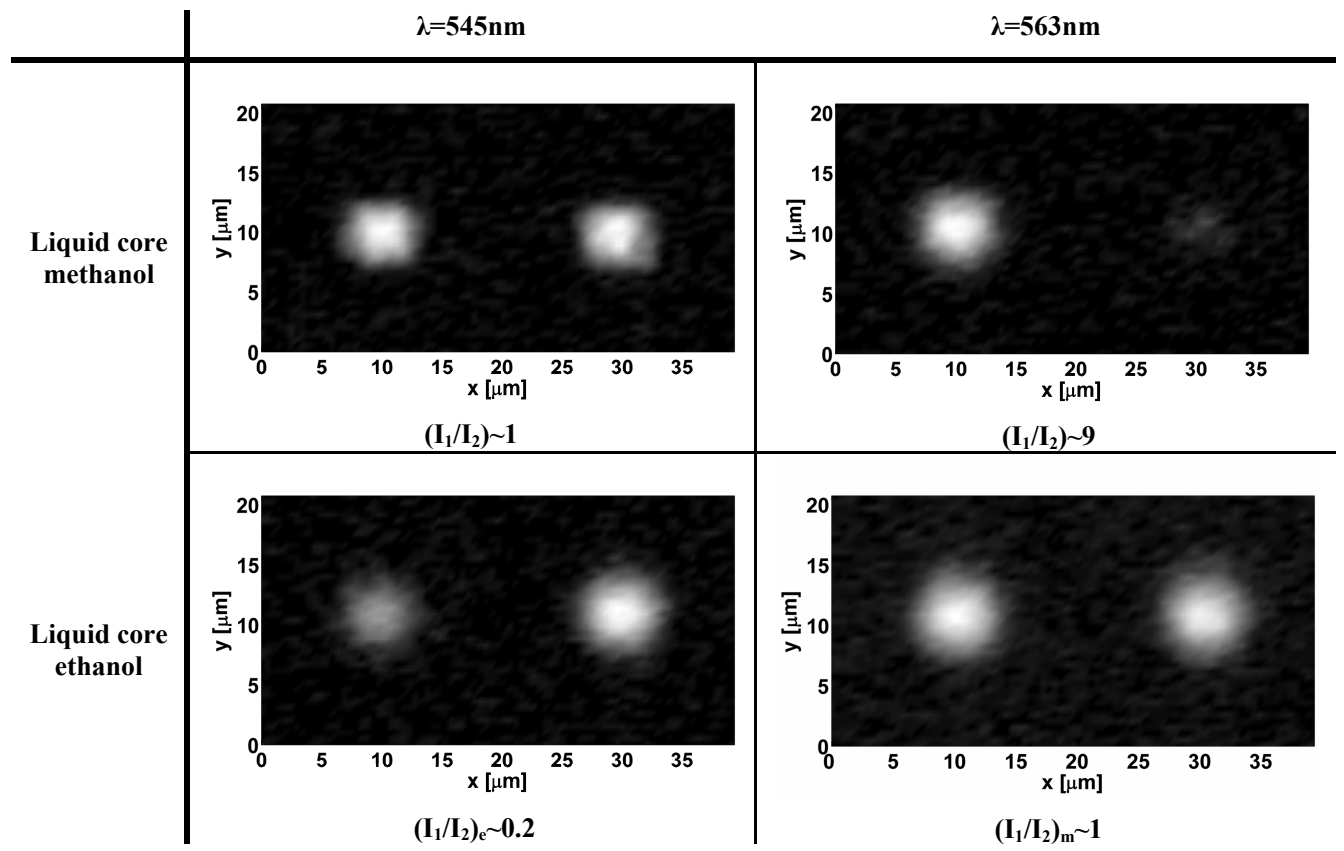

Fig. 7 CCD captured output intensity distribution from the OP1 and OP2 of the MMIm and MMIe at $\lambda=545 \mathrm{~nm}$ and $\lambda=563 \mathrm{~nm}$.

\section{2. $1^{\text {st }}$ generation optofluidic Mach-Zehnder interferometer}

In conventional integrated Mach-Zehnder interferometers (MZI), based on solid core waveguides, the phase shift is induced in one arm by a variation of effective refractive index of the waveguide [21-24]. In this approach the interaction between the light and surrounding liquid medium is related only to the evanescent part of the modal field. So, in order to get a substantial variation at the output of the MZI for high efficiency detection, it is necessary use nanodevices or very long interaction lengths $[22,24]$.

In our MZI configuration, based on ARROW waveguide, since the mode field is almost completely confined into the liquid core of the waveguide, the interaction of the sample and field is strongly enhanced as compared to solid core MZI [14]. In figure 8 a schematic layout of the optofluidic Mach-Zehnder interferometer is reported. Light is coupled into the first straight waveguide and then splits by a y-junction into the left and right arms. The y-junction is composed of two different cos-shaped bends both shaped to reduce the bend losses.

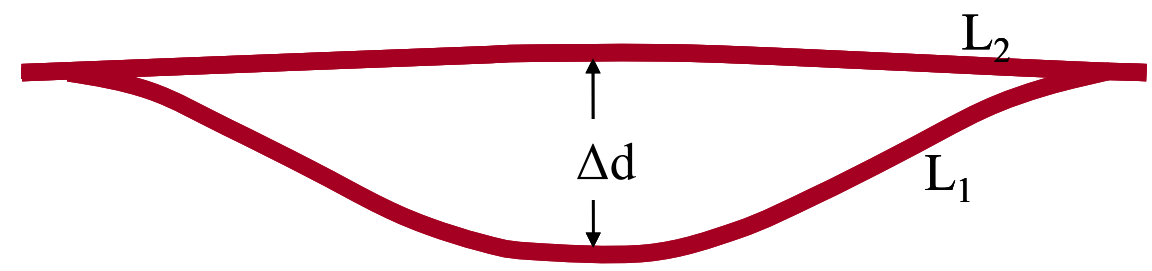

Fig. 8. Schematic layout of the optofluidic Mach Zhender interferometer.

The interferometer arms have a different optical length $\left(\mathrm{L}_{1}, \mathrm{~L}_{2}\right)$. For a fixed input wavelength $\lambda$, the phase difference accumulated at the output is 


$$
\Delta \varphi=\frac{2 \pi}{\lambda} \mathrm{n}_{\text {eff }}\left(\mathrm{L}_{1}-\mathrm{L}_{2}\right)
$$

Two asymmetric $\mathrm{MZ}$ configurations $\left(\mathrm{MZ}_{1}\right.$ and $\left.\mathrm{MZ}_{2}\right)$ were fabricated varying the maximum separation between arms and the y junction parameters. Fig.9 shows the SEM images of the y-junctions of the two MZ interferometers. The images illustrate the lower wafer before adding the upper wafer on top of it.

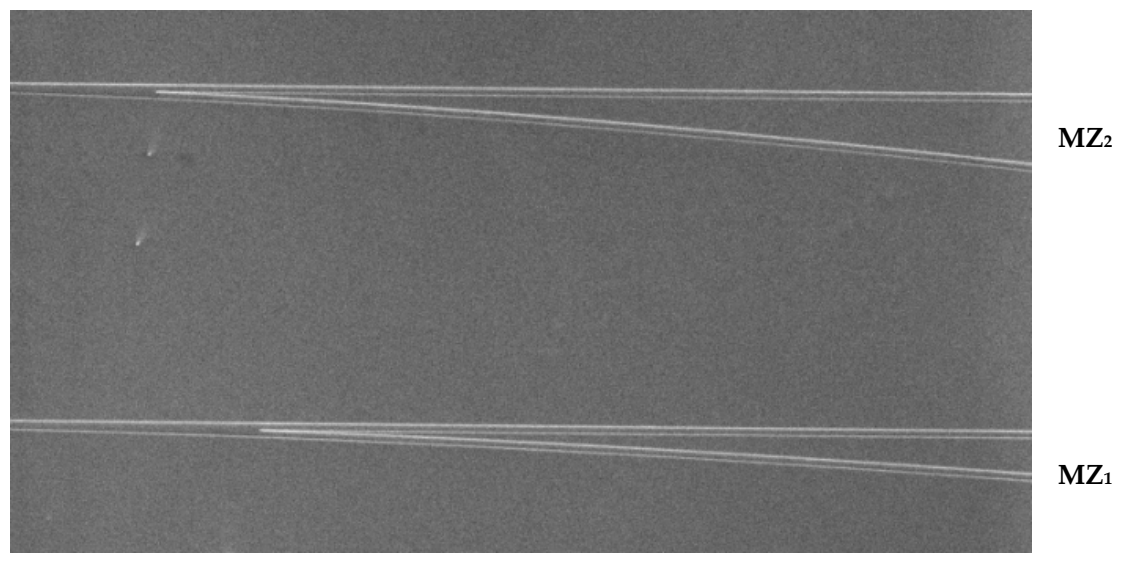

Fig. 9. SEM images of the y-junctions of the two MZ interferometers.

In the $\mathrm{MZ}_{1}$ configuration the maximum distance between the left and right branch is $\Delta \mathrm{d}=310 \mu \mathrm{m}$ and the bends radii are $\mathrm{R}_{1}=28.50 \mathrm{~mm}$ and $\mathrm{R}_{2}=85.6 \mathrm{~cm}$, respectively. Such an asymmetric configuration give rises to a length difference between the two arms of $\Delta \mathrm{L}=17.05 \mu \mathrm{m}$. The $\mathrm{y}$-junction parameters for the $\mathrm{MZ}_{2}$ configuration are $\Delta \mathrm{d}=510 \mu \mathrm{m}, \mathrm{R}_{1}=17.10 \mathrm{~mm}$, $\mathrm{R}_{2}=85.6 \mathrm{~cm}$ and $\Delta \mathrm{L}=47.30 \mu \mathrm{m}$. In both configurations the total length of the devices is $\mathrm{L}=15 \mathrm{~mm}$ and the required core volume is about $1.5 \mathrm{nl}$.

The devices was characterized using an unpolarized white lamp end-coupled into the waveguide core through an optical fiber with a $50 \mu \mathrm{m}$ core diameter. The transmitted light was collected with an optical fiber ( $50 \mu \mathrm{m}$ core) connected to a

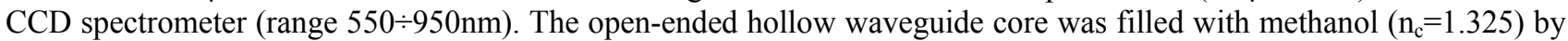
capillary effect due to the very small dimension of the device. For the precise alignment of all components a micrometer translation stage has been used.

The transmitted optical intensity at the output in a MZ interferometer is

$$
\mathrm{I} \propto 1+\mathrm{V} \cos (\Delta \varphi)
$$

where the phase difference $\Delta \varphi$ is given by eq.(1), $V=\left(\mathrm{I}_{\text {Max }}-\mathrm{I}_{\text {Min }}\right) /\left(\mathrm{I}_{\text {Max }}+\mathrm{I}_{\text {Min }}\right)$ is the visibility of the interferometer. In fig. 10 the spectra transmitted from the $\mathrm{MZ}_{1}$ and $\mathrm{MZ}_{2}$ are reported. The intensities are normalized to the maximum of the spectrum respectively.

The total loss from the input to the output of the devices is $42 \mathrm{~dB}$ for $\mathrm{MZ}_{1}$ and $45 \mathrm{~dB}$ for $\mathrm{MZ}_{2}$. The transmitted spectrum is the result of the spectral properties of the ARROW waveguide and the MZ interferometer. From these figures, we recognize the typical spectral behavior of an antiresonant reflecting optical waveguide with broad antiresonant peak centered around the design value $(\lambda=633 \mathrm{~nm})$ and the interference pattern of the Mach-Zehnder interferometer. From the measured transmitted spectrum we have evaluated a free-spectral range of $\mathrm{FSR}_{1} \approx 16.5 \mathrm{~nm}$ for the $\mathrm{MZ}_{1}$ and of $\mathrm{FSR}_{2} \approx 5.8 \mathrm{~nm}$ for the $\mathrm{MZ}_{2}$. These values are in good agreement with the expected free spectral range of $\mathrm{FSR}_{1} \approx 16 \mathrm{~nm}$ and $\mathrm{FSR}_{2} \approx 6 \mathrm{~nm}$.

The visibility measured from the spectra (i.e. the difference between maximum and minimum of the subsequent fringes in the interference pattern) is $\mathrm{V}_{1}=0.453$ for the $\mathrm{MZ}_{1}$ and $\mathrm{V}_{2}=0.375$ for the $\mathrm{MZ}_{2}$. The low visibility values depend on the strong intensity unbalance of the beams in each arm. This unbalance is essentially due to the different bend losses of the two arms. 


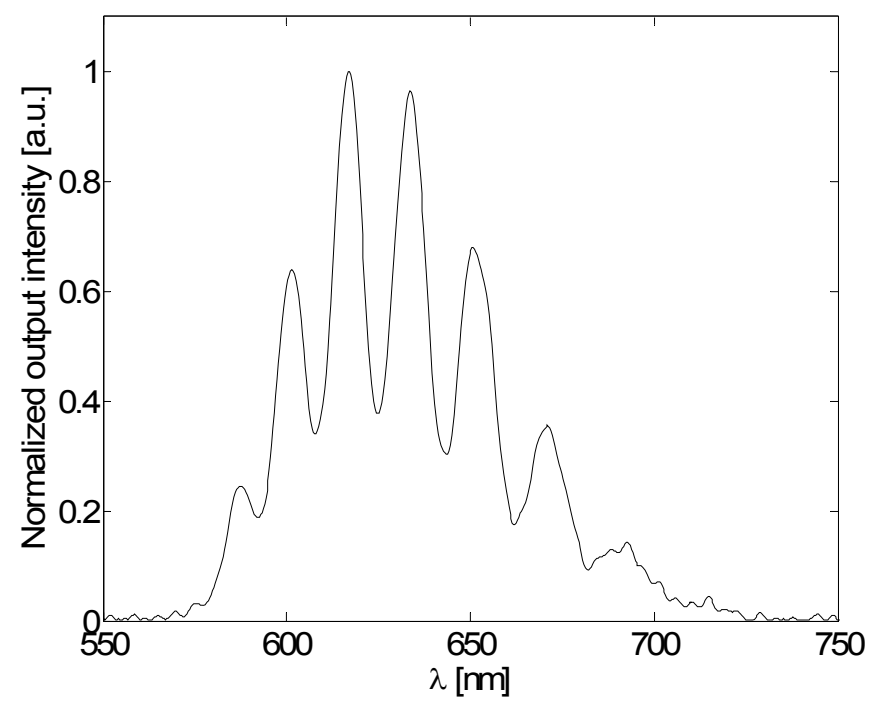

Fig.10 Measured interference pattern for the $\mathrm{Mz}_{1}(\mathrm{FSR} \approx 16.5 \mathrm{~nm})$.

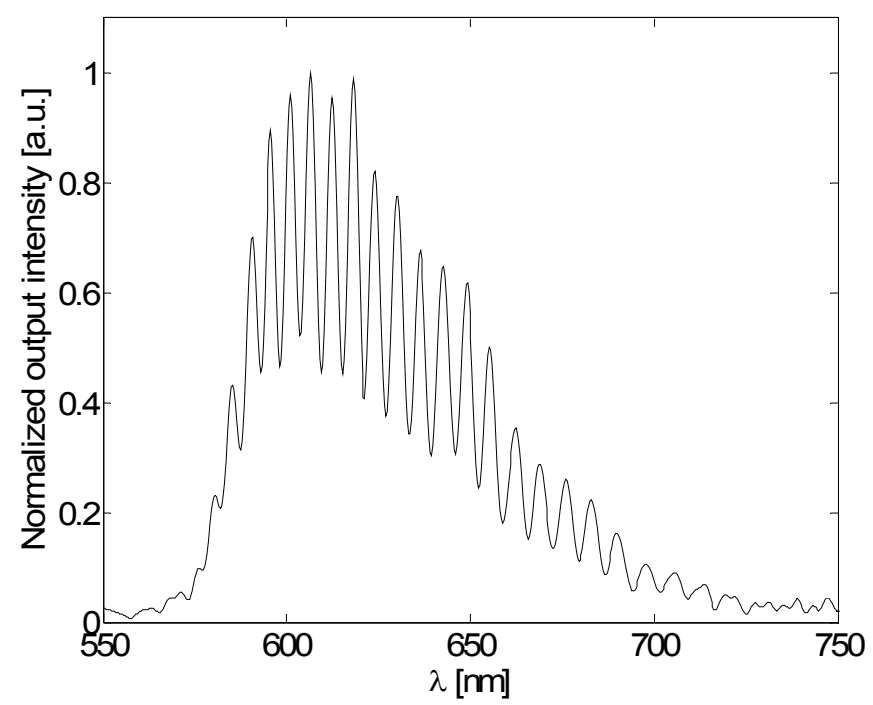

Fig.10 Measured interference pattern for the $\mathrm{Mz}_{2}(\mathrm{FSR} \approx 5.8 \mathrm{~nm})$.

\section{3. $2^{\text {st }}$ generation optofluidic Mach-Zehnder interferometer.}

In the $1^{\text {st }}$ generation of optofluidic MZI the device is realized by two y-junctions each one composed of two different cos-shaped bends [14]. However, as previously reported, this asymmetric geometry induces a strong intensity unbalance of the beam in each arm due to the different bend losses with a strong degradation of the device's performances due to the low visibility of the interferometer.

In order to overcome this problem, we have designed a novel geometry in order to minimize the intensity unbalance between the two arms also for high asymmetric Mach-Zehnder configurations. The device is based on T-branches and $90^{\circ}$-bent waveguides. This configuration permits to obtain very high visibility values with a strong improvements on the sensitivity of the device. 
A schematic layout of the MZI is reported in fig.11. Light is coupled into a straight waveguide followed by a taper with a starting width pf $10 \mu \mathrm{m}$ and a final width of $20 \mu \mathrm{m}$.The taper length is $500 \mu \mathrm{m}$. At the first T-branch the light is equally splitted into the upper and lower waveguides and recombined at the T-branch on the left. This configuration permits to realize a very compact device with a total length only $\mathrm{L}=2.5 \mathrm{~mm}$ and with required liquid volume is about $0.16 \mathrm{nl}$.

In this case the devices has been characterized using diode laser with a central wavelength of $660 \mathrm{~nm}$ coupled to a single mode fiber.

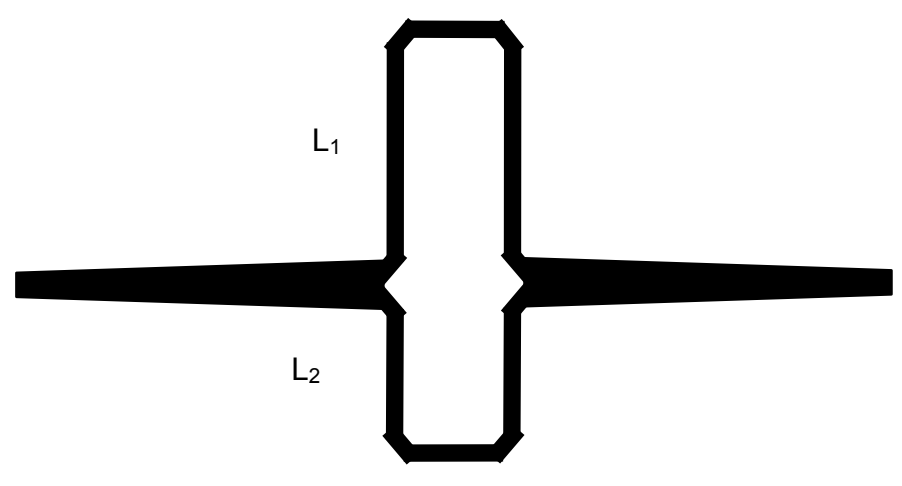

Fig.11 Schematic layout of the $2^{\text {st }}$ generation MZI.

In Fig. 12 is showed the measured transmitted spectra from the a MZI with a length difference $\Delta \mathrm{L}=200 \mu \mathrm{m}$ between the two arms. The free spectral range (FSR) measured from the spectra around $\lambda=660 \mathrm{~nm}$ is $1.57 \mathrm{~nm}$, and agrees well with the theoretical one $(1.60 \mathrm{~nm})$. Despite the MZI is strongly unbalanced, the measured visibility is 0.975 , meaning an great improvement compared to the $1^{\text {st }}$ generation MZI.

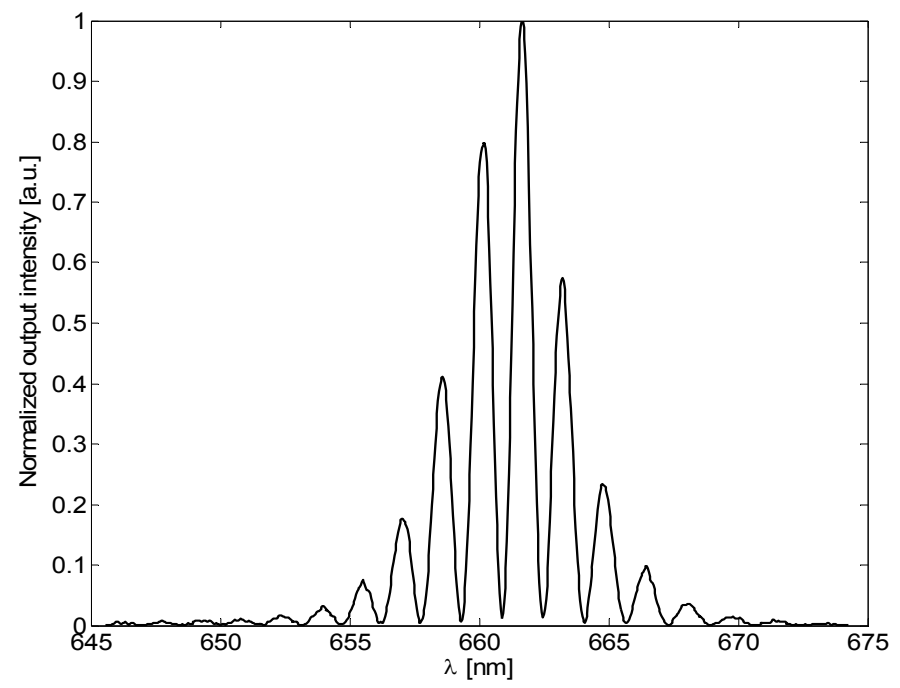

Fig. 12 Measured interference pattern for the $2^{\text {st }}$ generation MZI (FSR $\left.\approx 1.57 \mathrm{~nm}\right)$ 


\section{CONCLUSIONS}

Integrated liquid core ARROW waveguides are important components for optofluidic devices. Using silicon technology low loss ARROW waveguides have been fabricated also for single mode geometry. The feasibility of a optofluidic MMI coupler have been demonstrated. The coupling properties of the device can be tuned by changing the liquid waveguide core. Two novel Mach-Zehnder interferometer have proposed and realized using ARROW waveguide. The $2^{\text {st }}$ generation of optofluidic Mach-Zhender interferometer has shown very high performance that can be usefully employed in sensing applications

\section{ACKNOWLEDGEMENTS}

The authors would like to thank the IC Process Group of DIMES for technical assistance in the device fabrication.

\section{REFERENCES}

[1] D. Psaltis, S. R. Quake, C Yang, "Developing optofluidic technology through the fusion of microfluidics and optics", Nature 442, 381-386 (2007).

[2] H. Schmidt, A. R. Hawkins, “ Optofluidic waveguides: I. Concepts and implementations“, Microfluid Nanofluid 4, $3-16$ (2008).

[3] J. N. McMullin, R. Narendra and C:R. James, "Hollow metallic waveguides in Silicon V-grooves", IEEE Photon. Technol. Lett. 5, 1080-1082 (1993).

[4] A. Datta,; E. In-Yong; A. Dhar, P. Kuban, R. Manor, I. Ahmad, S. Gangopadhyay, T. Dallas, M. Holtz, H. Temkin, P.K. Dasgupta, "Microfabrication and characterization of teflon AF-coated liquid core waveguide channels in silicon", IEEE Sens. Journal 3, 788 - 795 (2003).

[5] S.S. Lo, M.S.Wang, C.C. Chen, "Semiconductor hollow optical waveguides formed by omni-directional reflectors", Optics Express 12, 6589-6593 (2004).

[6] A. Grosse, M. Grewe and H. Fouckhardt, "Deep wet etching of fused silica glass for hollow capillary optical leaky waveguides in microfluidic devices", J. Micromech. Microeng. 11, 257-262 (2001).

[7] T. Baba and Y. Kokubun, "Dispersion and radiation loss characteristics of antiresonant reflecting optical waveguides - numerical results and analytical expressions", IEEE J. Quantum. Electron. 28, 1689-1700 (1992).

[8] N.J. Goddard, K. Singh, F. Bounaria, R.J. Holmes, S.J. Baldock, L.W. Pickering, P.R. Fielden and R.D. Snook, "Anti-Resonant Reflecting Optical Waveguides (ARROWS) as Optimal Optical Detectors for MicroTAS Applications", in Proceedings of the $\mu T A S$ '98 Workshop, D.J. Harrison and A. Van Den Berg, ed. Kluwer, 97 (1998).

[9] T. Delonges and H. Fouckhardt, "Integrated optical detection cell based on Bragg reflecting waveguides", $J$. of Chromatography A 716, 135-139 (1995).

[10]R. Bernini, S. Campopiano, L. Zeni, "Silicon Micromachined Hollow Optical Waveguides for Sensing Applications", IEEE J. Selected Topics in Quantum Elect. 8, 106 (2002).

[11] S. Campopiano, R. Bernini, L. Zeni, P.M. Sarro, "Microfluidic sensor based on integrated optical hollow waveguide", Optics Lett. 29, 1894-1896 (2004).

[12] D.Yin, D.W Deamer, H.Schmidt, J.P. Barber, A.R.Hawkins, "Integrated optical waveguides with liquid cores", Appl. Phys. Lett,. 85, 3477-3479 (2004).

[13] G. Testa, Y. Huang, L. Zeni, P.M.Sarro, R. Bernini,"'Liquid core ARROW waveguides by Atomic Layer Deposition", IEEE Photon. Technol. Lett. in print , (2010).

[14] R. Bernini, G. Testa, L. Zeni, and P. M. Sarro, "Integrated optofluidic Mach-Zehnder interferometer based on liquid core waveguide", Appl. Phys. Lett. 93, 011106-011108 (2008).

[15]D. Yin, H. Schmidt, J. P. Barber, E. J. Lunt, A. R. Hawkins, "Optical characterization of arch-shaped ARROW waveguides with liquid cores", Optics Express 13, 10564 - 10570 (2005).

[16] M. Leskela, M. Ritala, "Atomic Layer Deposition Chemistry: Recent Developments and Future Challenges", Angew. Chem. Int. 42, 5548-5554 (2003).

[17] L. Soldano and E. Pennings, "Optical multi-mode interference devices based on self-imaging: Principles and applications," J. Lightw. Technol. 13, 615-627 (1995).

[18] J. Leuthol and C. H. Joyner, "Multimode Interference Couplers with Tunable Power Splitting Ratios", J. Lightwave Technol. 19, 700-707 (2001). 
[19] C. H. Bae and F. Koyama, "Fabrication and characterization of hollow waveguide optical switch with variable air core", Optics Express 13, 3259-3263 (2005).

[20] R. Bernini, E. De Nuccio, A. Minardo, L. Zeni and P. M. Sarro, "2-D MMI Devices Based on Integrated Hollow ARROW Waveguides”, IEEE J. Sel. Topics Quantum Electron, 13, 194-201 (2007).

[21] William M. Green, Michael J. Rooks, Lidija Sekaric, and Yurii A. Vlasov, "Ultra-compact, low RF power, $10 \mathrm{~Gb} / \mathrm{s}$ silicon Mach-Zehnder modulator," Opt. Express 15, 17106-17113 (2007)

[22]F. Prieto, B.Sepúlveda, A. Calle, A. Llobera, C. Domínguez, A. Abad, A. Montoya, and L. M. Lechuga, "An integrated optical interferometric nanodevice based on silicon technology for biosensor applications", Nanotechnology, 14, 907-912 (2003).

[23] S. Y. Wu, H. P. Ho, W. C. Law, Chinlon Lin, and S. K. Kong, "Highly sensitive differential phase-sensitive surface plasmon resonance biosensor based on the Mach-Zehnder configuration," Opt. Lett. 29, 2378-2380 (2004).

[24]A. Densmore, D.-X. Xu, S. Janz, P. Waldron, T. Mischki, G. Lopinski, A. Delâge, J. Lapointe, P. Cheben, B. Lamontagne, and J. H. Schmid, "Spiral-path high-sensitivity silicon photonic wire molecular sensor with temperature-independent response," Opt. Lett. 33, 596-598 (2008). 